\title{
Optimal Relay Selection for Physical Layer Security with Cooperative Jamming
}

\author{
Lijun Jia, Yucheng He \\ College of Information Science and Engineering, Huaqiao University, 668 Jimei Avenue, Xiamen, China \\ \{f_author, s_author\}1342562554@qq.com, he_yucheng@163.com
}

Keywords: relay selection, physical layer security, cooperative jamming, secrecy outage probability (SOP)

\begin{abstract}
This paper studies a scheme of optimal relay selection with cooperative jamming and optimal power allocation factor between forwarding relay and jammer. The traditional optimal relay selection scheme selects an optimal relay node to assist forward source information. Based on legitimate user and eavesdropper instantaneous channel state information, the proposed scheme selects two relay nodes to increase security against eavesdropper. The first relays to forward source information as traditional scheme. The second relays to send artificial noise as a friendly jammer. The relay operates in Decode-and-Forward strategy. The closed form expression of secrecy outage probability is derived. In addition, the optimal power allocation factor is given when the total relay transmit power is constrained. The simulation results verify that the proposed scheme can significantly improve system security performance compared with the traditional scheme.
\end{abstract}

\section{INTRODUCTION}

The security and privacy have more and more attention with the wide application of wireless communication technology. The purpose of secure communication is that legitimate receivers can successfully receive the source information and the eavesdropper fail to intercept the information. The physical layer security makes full use of the various communication characteristics of the wireless channel to solve the communication process information security issues from the information theory point of view.

The traditional information security problem is solved by the higher layers protocol and encryption algorithm based on the key system. However, it is hard to overcome the negative effects due to broadcast characteristics of wireless channels and the rapidly increase for computing power on information security. The theoretical basis of the physical layer security is Shannon's perfect secrecy theory. Wyner proposed the concept of the physical layer security(A. D.Wyner, 1975) . It is found that a new security mechanism can achieve "Absolute security" which do not depend on traditional encryption algorithm rather than using the natural physical characteristics of the wireless channel.the physical layer security is extended to the Gaussian Wiretap Channel(S. Leung-Yan-Cheong, 1978). The confidential signal transmission in the wireless broadcast channel is further studied(I. Csiszar, 1978).

However, the secure transmission rate is greatly influenced by the channel conditions. When the channel condition of the legitimate user is similar to the eavesdropping user, security transmission rate may be very low or even zero. In order to solve this problem, the optimal relay beamforming algorithm under multi-relay scenes was studied(L. Dong, 2010). The impact of the system on the security performance was studied when the channel correlation fading coefficient and the selected relay may be outdated(L. Fan, 2017). The opportunistic relay selection in a secure network with secure constraints(I. Krikidis, 2010). The physical layer security scheme based on cooperative interference in the energy cooperative network(Weijia Lei, 2015). In order to improve the secrecy rate, the relay node sends the beamforming interference signal to interfere eavesdropper. The problem of secure transmission based on cooperative interference with multiple eavesdroppers was studied(L. Tang, 2016). A physical layer hybrid transmission protocol was proposed based on cooperative interference for the security problem of full duplex relay system(Wei Zhou, 2017). The optimal relay selection is studied in secure cooperative communication with an adaptive eavesdropper(L. Yang, 2017). Joint relay and jammer select in a relay cooperative network is studied(I. Krikidis, 2009). The scheme assumes that all relay nodes can correctly decode the source signal during the broadcast phase. The channel state information of the first hop link is ignored.

On the basis of the above research, a joint relay and jammer selection based on instantaneous channel state information for all links is studied. Optimal power allocation factor is given to maximize the system secrecy capacity. Performance analysis is compared with traditional optimal relay selection scheme. The systems secrecy outage probability (SOP) is obtained after selection criteria of two schemes.

\section{SYSTEM MODEL}

As shown in Figure 1, a physical layer secure communication system model with decode-forward and cooperative interference protocol (DF / CJ) is considered. The model contains a pair of nodes with legitimate source node $\mathrm{S}$ and receiver $\mathrm{D}$, an eavesdropper $\mathrm{E}$ and relay set $R_{\mathrm{SR}}=\left\{\mathrm{R}_{k}\right\}_{k=1}^{K}$ with $\mathrm{K}$ relays. We assume that the direct links $(S \rightarrow D, S \rightarrow E)$ are not available. All nodes work in half-duplex mode, they cannot transmit and receive simultaneously. Each communication process between source and the destination is divided into two phases: the first phase, the source node 
broadcasts messages to all relay nodes; the second phase, two selected relays transmit massage towards the destination and the eavesdropper. The first relay $\mathrm{R}$ is selected (as shown in the figure 1 with $\mathrm{R}_{i}$ ) to forward source message, the second relay $\mathrm{J}$ operates a "jammer mode" (as shown in the figure 1 with $\mathrm{R}_{j}$ ) to the interference eavesdropper.

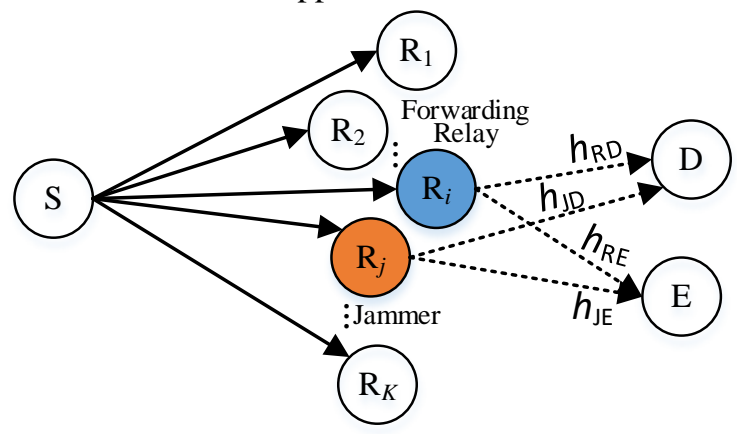

Figure 1: System model

A slow, flat, block Rayleigh fading environment is assumed and the full channel state information is known, where the channel is independently. $P_{i}$ denotes the transmitted power for $i$-th node, $h_{i j}$ is the channel coefficient for the link $i \rightarrow j$ modeled as a zero-mean, independent, circularly-symmetric complex Gaussian random variable with variance $1, \gamma_{i j}=P_{i}\left|h_{i j}\right|^{2}$ is the instantaneous signal-to-noise ratio (SNR) for the link $i \rightarrow j$.

In the first stage, the mutual information between the source node and relay node as

$$
C_{\mathrm{SR}_{k}}=\frac{1}{2} \log _{2}\left(1+\gamma_{\mathrm{SR}_{k}}\right)
$$

Where factor $1 / 2$ is due to the transmission process is divided into two time slots.

In adaptive DF relay protocol, $R_{\mathrm{tr}}$ denotes system information transmission rate. When $C_{S R_{k}}>R_{\mathrm{tr}}$, it is assumed the relays can successfully decode the source signal which forms a decoding set $R_{\mathrm{DF}},\left|R_{\mathrm{DF}}\right|$ represents the number of relays contained in $R_{\mathrm{DF}}$. We note that although the node $R$ must belong to the decoding set $R_{\mathrm{DF}}$ due to the DF protocol, the node $J$ can be any node of the relay set $R_{\mathrm{SR}}$ as it does not require to decode the source message. Thus, the instantaneous secrecy capacity of the system is defined as

$$
C_{S}=\left[\frac{1}{2} \log _{2}\left(1+\frac{\gamma_{\mathrm{RD}}}{1+\gamma_{\mathrm{JD}}}\right)-\frac{1}{2} \log _{2}\left(1+\frac{\gamma_{\mathrm{RE}}}{1+\gamma_{\mathrm{JE}}}\right)\right]^{+}
$$

Where $[x]^{+} \triangleq \max \{0, x\}$.

Assumed $R_{S}$ is the system requires the minimum secrecy transmission rate, when the system secrecy capacity $C_{S}$ is less than $R_{S}, S$ to $\mathrm{D}$ information transmission is interrupted. Therefore, the secrecy outage probability is an important index of the systems secrecy transmission performance. In this paper, the systems secrecy outage probability (SOP) is define as a function of $R_{S}$

$$
\begin{aligned}
& P_{\text {out }}\left(R_{S}\right) \triangleq \operatorname{Pr}\left\{C_{S}<R_{S}\right\} \\
& =\sum_{N=0}^{K} \operatorname{Pr}\left\{\left|R_{\mathrm{DF}}\right|=N\right\} \operatorname{Pr}\left\{C_{S}<R_{S}|| R_{\mathrm{DF}} \mid=N\right\}
\end{aligned}
$$

With

$$
\begin{aligned}
& \operatorname{Pr}\left\{\left|R_{\mathrm{DF}}\right|=N\right\}=\left(\begin{array}{l}
K \\
N
\end{array}\right)\left[\operatorname{Pr}\left(C_{S R_{k}}>R_{T}\right)\right]^{N} \\
& \times\left[\operatorname{Pr}\left(C_{S R_{k}} \leq R_{T}\right)\right]^{K-N}
\end{aligned}
$$

\section{JOINT RELAY AND JAMMER SELECT SCHEME}

In this section, we first discuss the optimal relay selection and the system secrecy outage probability without jamming. Then we consider optimal relay selection with jamming and give the optimal power distribution factor.

\subsection{Optimal relay selection}

When cooperative interference is not considered, an optimal relay node $\mathrm{R}$ is selected to forward source massage. Communication in fading environment usually satisfy $\gamma_{\mathrm{RD}} \gg 1$ and $\gamma_{\mathrm{RE}} \gg 1$. Thus, the instantaneous reachable secrecy capacity of the system is approximated by formula (2) as

$$
C_{S}=\left[\frac{1}{2} \log _{2}\left(\frac{1+\gamma_{\mathrm{RD}}}{1+\gamma_{\mathrm{RE}}}\right)\right]^{+} \simeq\left[\frac{1}{2} \log _{2}\left(\frac{\gamma_{\mathrm{RD}}}{\gamma_{\mathrm{RE}}}\right)\right]^{+}
$$

Due to the presence of eavesdropper, the optimal relay selection should maximize the received signal to noise ratio of the forwarding channel, and the received signal to noise ratio of the wiretap channel should minimize simultaneously, that is the relay selection criterion should maximize the system secrecy capacity. Thus, when the all channel state information is known, the expression of the optimal relay selection criterion is obtained from equation (5) as

$$
\mathrm{R}=\underset{\mathrm{R}_{i}}{\arg \max }\left\{\frac{\gamma_{\mathrm{R}_{i} \mathrm{D}}}{\gamma_{\mathrm{R}_{i} \mathrm{E}}}\right\}=\underset{\mathrm{R}_{i}}{\arg \max }\left\{\frac{\left|h_{\mathrm{R}_{i} \mathrm{D}}\right|^{2}}{\left|h_{\mathrm{R}_{i} \mathrm{E}}\right|^{2}}\right\}
$$

Substituting (5) into (3), the system secrecy outage probability is given as

$$
\begin{gathered}
P_{\text {out }}\left(R_{S}\right)=\sum_{N=0}^{K} \operatorname{Pr}\left\{\left|R_{\mathrm{DF}}\right|=N\right\} \operatorname{Pr}\left\{C_{S}<R_{S}|| R_{\mathrm{DF}} \mid=N\right\} \\
\operatorname{Pr}\left\{C_{S}<R_{S}|| R_{\mathrm{DF}} \mid=N\right\} \simeq \operatorname{Pr}\left\{\frac{\left|h_{\mathrm{RD}}\right|^{2}}{\left|h_{\mathrm{RE}}\right|^{2}}<2^{2 R_{S}}\right\}
\end{gathered}
$$


Where $\operatorname{Pr}\left\{\left|R_{\mathrm{DF}}\right|=N\right\}$ denotes the probability of relay number is $\mathrm{N}$ in the set $R_{\mathrm{DF}}$. From (1) and (4) as

$$
\begin{aligned}
& \operatorname{Pr}\left\{\left|R_{\mathrm{DF}}\right|=N\right\}=\left(\begin{array}{c}
K \\
N
\end{array}\right)\left[\operatorname{Pr}\left(C_{S R_{k}}>R_{T}\right)\right]^{N} \\
& \times\left[\operatorname{Pr}\left(C_{S R_{k}} \leq R_{T}\right)\right]^{K-N}=\left(\begin{array}{c}
K \\
N
\end{array}\right) \times \\
& {\left[\exp \left(-\frac{2^{2 \mathrm{R}_{\mathrm{T}}}-1}{\bar{\gamma}_{\mathrm{SR}}}\right)\right]^{N}\left[1-\exp \left(-\frac{2^{2 \mathrm{R}_{\mathrm{T}}}-1}{\bar{\gamma}_{\mathrm{SR}}}\right)\right]^{K-N}}
\end{aligned}
$$

If $N=0$, then no relay will be selected so that the information transmission from $\mathrm{S}$ to $\mathrm{D}$ is interrupted. Therefore $\operatorname{Pr}\left\{C_{S}<R_{S} \| R_{\mathrm{DF}} \mid=N\right\}=1$.

If $N>0$, relay selection according to equation (6), then $\operatorname{Pr}\left\{C_{S}<R_{S} \| R_{\mathrm{DF}} \mid=N\right\}$ denotes outage probability after the relay node $\mathrm{R}$ is selected from set $R_{\mathrm{DF}}$, which is derived from theorem 1 .

Theorem 1: The outage probability of the relay node $\mathrm{R}$ is selected from set $R_{\mathrm{DF}}$ as

$$
\operatorname{Pr}\left\{C_{S}<R_{S}|| R_{\mathrm{DF}} \mid=N\right\} \simeq \prod_{i=1}^{N}\left(\frac{\mu}{\mu+\lambda_{i}}\right)
$$

Where $\quad \mu=2^{2 R_{S}} \quad, \quad \lambda_{i}=\mathbb{E}\left[\left|h_{\mathrm{R}_{i} \mathrm{D}}\right|^{2}\right] / \mathbb{E}\left[\left|h_{\mathrm{R}_{i} \mathrm{E}}\right|^{2}\right]$ represents the ratio of the average channel gain $\mathrm{R}_{i} \rightarrow \mathrm{D}$ and $\mathrm{R}_{i} \rightarrow \mathrm{E}$.

Proof: Define random variables $X_{i} \triangleq\left|h_{\mathrm{R}_{i} \mathrm{D}}\right|^{2}>0$, $Y_{i} \triangleq\left|h_{\mathrm{R}_{i} \mathrm{E}}\right|^{2}>0, \quad Z_{i} \triangleq X_{i} / Y_{i}$. Since $h_{\mathrm{R}_{i} \mathrm{D}}$ and $h_{\mathrm{R}_{i} \mathrm{E}}$ are independently of each other, therefore $X_{i}$ and $Y_{i}$ are also independently, the probability distribution function (CDF) of the random variable $Z_{i}$ can be deduced as follows:

$$
\begin{aligned}
& F_{Z_{i}}(z)=\operatorname{Pr}\left\{Z_{i}<z\right\}=\operatorname{Pr}\left\{\frac{X_{i}}{Y_{i}}<z\right\} \\
& =\operatorname{Pr}\left\{X_{i}<z Y_{i}\right\}=\int_{0}^{\infty} F_{X_{i}}(z y) f_{Y_{i}}(y) d y
\end{aligned}
$$

According to the channel model, both $X_{i}$ and $Y_{i}$ obey the exponential distribution. The probability distribution function (CDF) and the probability density function (PDF) are given respectively as follows:

$$
\begin{aligned}
& F_{X_{i}}(x)=1-\exp \left(-\frac{x}{\theta_{X_{i}}}\right), \quad x>0 \\
& f_{Y_{i}}(y)=\frac{1}{\theta_{Y_{i}}} \exp \left(-\frac{y}{\theta_{Y_{i}}}\right), \quad y>0
\end{aligned}
$$

Where parameters are $\theta_{X_{i}}=\mathbb{E}\left[\left|h_{\mathrm{R}_{i} \mathrm{D}}\right|^{2}\right]$ and
$\theta_{Y_{i}}=\mathbb{E}\left[\left|h_{\mathrm{R}_{i} \mathrm{E}}\right|^{2}\right]$. Substituting (12) and (13) into (11):

$$
F_{Z_{i}}(z)=\frac{z}{Z+\theta_{X_{i}} / \theta_{Y_{i}}}
$$

Define random variables $Z \triangleq \max _{i=1,2, \cdots, N}\left\{Z_{i}\right\}$, According to the channel model we can see random variables $Z_{i}(i=1,2, \cdots, N)$ independently of each other, The probability distribution function (CDF) of $\mathrm{Z}$ as

$$
F_{Z}(z)=\prod_{i=1}^{N}\left(\frac{z}{Z+\theta_{X_{i}} / \theta_{Y_{i}}}\right)
$$

From (8) and (15), we can get the conclusion (10).

Substituting (9) and (10) into (7), the system secrecy outage probability of optimal relay selection can be expressed as

$$
\begin{aligned}
& P_{\text {out }}\left(R_{S}\right)=\sum_{N=0}^{K}\left(\begin{array}{c}
K \\
N
\end{array}\right)\left[\exp \left(-\frac{2^{2 \mathrm{R}_{\mathrm{T}}}-1}{\bar{\gamma}_{\mathrm{SR}}}\right)\right]^{N} \\
& \times\left[1-\exp \left(-\frac{2^{2 \mathrm{R}_{\mathrm{T}}}-1}{\bar{\gamma}_{\mathrm{SR}}}\right)\right]^{K-N}\left[\prod_{i=1}^{N}\left(\frac{\mu}{\mu+\lambda_{i}}\right)\right]
\end{aligned}
$$

Where $\mu=2^{2 R_{S}}$.

\subsection{Optimal relay selection with jamming}

The joint relay and jammer selection is discussed below. First relay forwarding node $\mathrm{R}$ is selected in the decoding set $R_{\mathrm{DF}}$ which represents the set of all relay nodes that can be correctly decoded, then jammer node $\mathrm{J}$ is selected in the set $R_{\mathrm{SR}}=\left\{\mathrm{R}_{k}\right\}_{k=1}^{K-1}$. Forwarding and jammer nodes are selected to maximum instantaneous secrecy capacity. When $R_{\mathrm{DF}}$ is not empty, according to the formula (2), the selection problem of forwarding node $\mathrm{R}$ and the jammer node $\mathrm{J}$ can be expressed as

$$
(\mathrm{R}, \mathrm{J})=\underset{\substack{\mathrm{R}_{i} \in R_{\mathrm{DF}} \\ \mathrm{R}_{j} \in R_{\mathrm{SR}} \\ \mathrm{R} \neq \mathrm{J}}}{\arg \max }\left\{C_{S}\right\}=\underset{\substack{\mathrm{R}_{i} \in R_{\mathrm{P}} \\ \mathrm{R}_{j} \in R_{\mathrm{SR}} \\ \mathrm{R} \neq \mathrm{J}}}{\arg \max }\left\{\frac{1+\frac{\gamma_{\mathrm{R}_{i} \mathrm{D}}}{1+\gamma_{\mathrm{R}_{j} \mathrm{D}}}}{1+\frac{\gamma_{\mathrm{R}_{i} \mathrm{E}}}{1+\gamma_{\mathrm{R}_{j} \mathrm{E}}}}\right\}
$$

It can be seen from equation (17) that the computational complexity of the selection criterion increases exponentially with the relay increase. To reduce the computational complexity, it can usually be assumed $\gamma_{\mathrm{R}_{j} \mathrm{D}} \gg 1, \gamma_{\mathrm{R}_{j} \mathrm{E}} \gg 1, P_{i} / P_{j} \gg 1$, the selection criterion can be approximated as

$$
(\mathrm{R}, \mathrm{J}) \simeq \underset{\substack{\mathrm{R}_{i} \in R_{\mathrm{DF}} \\
\mathrm{R}_{\mathrm{R}} \in R_{\mathrm{SR}} \\
\mathrm{R} \neq \mathrm{J}}}{\operatorname{argmax}}\left\{\frac{\frac{\left|h_{\mathrm{R}_{i} \mathrm{D}}\right|^{2}}{\left|h_{\mathrm{R}_{j}}\right|^{2}}}{\mid \frac{\left.h_{\mathrm{R}_{i} \mathrm{E}}\right|^{2}}{\left|h_{\mathrm{R}_{j}}\right|^{2}}}\right\} \Rightarrow\left\{\begin{array}{l}
\mathrm{R}=\underset{\mathrm{R}_{i} \in R_{\mathrm{DF}}}{\arg \max }\left\{\frac{\left|h_{\mathrm{R}_{i} \mathrm{D}}\right|^{2}}{\left|h_{\mathrm{R}_{i} \mathrm{E}}\right|^{2}}\right\} \\
\mathrm{J}=\underset{\mathrm{R}_{j} \in R_{\mathrm{SR}}}{\arg \min }\left\{\frac{\left|h_{\mathrm{R}_{j} \mathrm{D}}\right|^{2}}{\left|h_{\mathrm{R}_{j} \mathrm{E}}\right|^{2}}\right\}
\end{array}\right.
$$

Substituting (2) into (3), the system secrecy outage 
probability is given as

$$
\begin{gathered}
P_{\text {out }}\left(R_{S}\right)=\sum_{N=0}^{K} \operatorname{Pr}\left\{\left|R_{\mathrm{DF}}\right|=N\right\} \operatorname{Pr}\left\{C_{S}<R_{S}|| R_{\mathrm{DF}} \mid=N\right\} \\
\operatorname{Pr}\left\{C_{S}<R_{S}|| R_{\mathrm{DF}} \mid=N\right\} \simeq \operatorname{Pr}\left\{\frac{1}{2} \log _{2}\left(\frac{\left|h_{\mathrm{RD}}\right|^{2}\left|h_{\mathrm{JE}}\right|^{2}}{\left|h_{\mathrm{RE}}\right|^{2}\left|h_{\mathrm{JD}}\right|^{2}}\right)<R_{S}\right\}
\end{gathered}
$$

Where $\operatorname{Pr}\left\{\left|R_{\mathrm{DF}}\right|=N\right\}$ denotes the probability that the number of relay nodes in the set $R_{\mathrm{DF}}$ is $\mathrm{N}$, from (9) we can know:

$$
\begin{aligned}
& \operatorname{Pr}\left\{\left|R_{\mathrm{DF}}\right|=N\right\}=\left(\begin{array}{c}
K \\
N
\end{array}\right)\left[\exp \left(-\frac{2^{2 \mathrm{R}_{\mathrm{T}}}-1}{\bar{\gamma}_{\mathrm{SR}}}\right)\right]^{N} \\
& \times\left[1-\exp \left(-\frac{2^{2 \mathrm{R}_{\mathrm{T}}}-1}{\bar{\gamma}_{\mathrm{SR}}}\right)\right]^{K-N}
\end{aligned}
$$

If $N=0$, then no relay will be selected so that the information transmission from $\mathrm{S}$ to $\mathrm{D}$ is interrupted. $\operatorname{Pr}\left\{C_{S}<R_{S} \| R_{\mathrm{DF}} \mid=N\right\}=1$.

If $N>0$, relay and jammer are selected according to (18), $\operatorname{Pr}\left\{C_{S}<R_{S}|| R_{\mathrm{DF}} \mid=N\right\}$ denotes outage probability after forwarding $\mathrm{R}$ and jammer $\mathrm{J}$ are selected, which is derived from theorem 2 .

Theorem 2: System outage probability after the forwarding node $\mathrm{R}$ and jammer $\mathrm{J}$ is selected as

$$
\begin{aligned}
& \operatorname{Pr}\left\{C_{S}<R_{S}|| R_{\mathrm{DF}} \mid=N\right\} \simeq \int_{0}^{\infty}\left[\prod_{i=1}^{N}\left(\frac{w_{2} \mu}{w_{2} \mu+\lambda_{i}}\right)\right] \\
& \times \frac{\left\{\frac{d}{d w_{2}}\left[\prod_{j=1}^{K-1}\left(w_{2}+\lambda_{j}\right)\right]\right\} \prod_{j=1}^{K-1} \lambda_{j}}{\prod_{j=1}^{K-1}\left(w_{2}+\lambda_{j}\right)^{2}} d w_{2}
\end{aligned}
$$

Where $\quad \mu=2^{2 R_{S}} \quad, \quad \lambda_{i}=\mathbb{E}\left[\left|h_{\mathrm{R}_{i} \mathrm{D}}\right|^{2}\right] / \mathbb{E}\left[\left|h_{\mathrm{R}_{i} \mathrm{E}}\right|^{2}\right]$ represents the ratio of the average channel gain $\mathrm{R}_{i} \rightarrow \mathrm{D}$ and $\mathrm{R}_{i} \rightarrow \mathrm{E}, \lambda_{j}=\mathbb{E}\left[\left|h_{\mathrm{R}_{j} \mathrm{D}}\right|^{2}\right] / \mathbb{E}\left[\left|h_{\mathrm{R}_{j} \mathrm{E}}\right|^{2}\right]$ represents the ratio of the average channel gain $\mathrm{R}_{j} \rightarrow \mathrm{D}$ and $\mathrm{R}_{j} \rightarrow \mathrm{E}$.

Proof: define random variables

$$
\mathrm{Z}_{i} \triangleq\left|h_{\mathrm{R}_{i} \mathrm{D}}\right|^{2} /\left|h_{\mathrm{R}_{i} \mathrm{E}}\right|^{2}, \mathrm{Z}_{j} \triangleq\left|h_{\mathrm{R}_{j} \mathrm{D}}\right|^{2} /\left|h_{\mathrm{R}_{j} \mathrm{E}}\right|^{2}(i, j \in\{1,2, \cdots, K\})
$$

the probability distribution function (CDF) of the random variable $Z_{j}$ is given by theorem 1 .

$$
F_{Z_{j}}(z)=\frac{z}{z+\lambda_{j}}
$$

Define random variables

$$
\begin{aligned}
& \mathrm{W}_{1} \triangleq \max \left\{\mathrm{Z}_{i}\right\}=\left|h_{\mathrm{RD}}\right|^{2} /\left|h_{\mathrm{RE}}\right|^{2} \\
& \mathrm{~W}_{2} \triangleq \min \left\{\mathrm{Z}_{j}\right\}=\left|h_{\mathrm{JD}}\right|^{2} /\left|h_{\mathrm{JE}}\right|^{2}
\end{aligned}
$$

Substituting $\mathrm{W}_{1}, \mathrm{~W}_{2}$ into (20) as

$$
\begin{aligned}
& P_{\text {out }} \simeq \operatorname{Pr}\left\{\frac{\mathrm{W}_{1}}{\mathrm{~W}_{2}}<2^{2 R_{S}}\right\}=\operatorname{Pr}\left\{\mathrm{W}_{1}<\mathrm{W}_{2} \mu\right\} \\
& =\int_{0}^{\infty} F_{\mathrm{W}_{1}}\left(w_{2} \mu\right) f_{\mathrm{W}_{2}}\left(w_{2}\right) d w_{2}
\end{aligned}
$$

The probability distribution function (CDF) of the random variable $\mathrm{W}_{1}$ from theorem 1 as

$$
F_{\mathrm{W}_{1}}\left(w_{1}\right)=\prod_{i=1}^{N}\left(\frac{w_{1}}{w_{1}+\lambda_{i}}\right)
$$

According to the channel model, we can know that the random variables $Z_{j}$ are independently of each other, the probability distribution function (CDF) of the random variable $\mathrm{W}_{2}$ is given (A. Papoulis, 2002).

$$
F_{\mathrm{W}_{2}}\left(w_{2}\right)=1-\prod_{j=1}^{K-1}\left(1-\frac{w_{2}}{w_{2}+\lambda_{j}}\right)
$$

The probability density function (PDF) of the random variable $\mathrm{W}_{2}$ is derived from (26)

$$
\frac{\left\{\frac{d}{d w_{2}}\left[\prod_{j=1}^{K-1}\left(w_{2}+\lambda_{j}\right)\right]\right\} \prod_{j=1}^{K-1} \lambda_{j}}{\prod_{j=1}^{K-1}\left(w_{2}+\lambda_{j}\right)^{2}}
$$
(22).

From (25), (27) and (24), we can get the conclusion

Substituting (21) and (22) into (19), system secrecy outage probability of optimal relay selection with jamming can be expressed as

$$
\begin{aligned}
& P_{\text {out }}\left(R_{\mathrm{s}}\right)=\sum_{N=0}^{K}\left(\begin{array}{l}
K \\
N
\end{array}\right)\left[\exp \left(-\frac{2^{2 \mathrm{R}_{\mathrm{T}}}-1}{\bar{\gamma}_{\mathrm{SR}}}\right)\right]^{N} \times \\
& {\left[1-\exp \left(-\frac{2^{2 \mathrm{R}_{\mathrm{T}}}-1}{\bar{\gamma}_{\mathrm{SR}}}\right)\right]^{K-N} \times \int_{0}^{\infty}\left[\prod_{i=1}^{N}\left(\frac{w_{2} \mu}{w_{2} \mu+\lambda_{i}}\right)\right]} \\
& \times \frac{\left\{\frac{d}{d w_{2}}\left[\prod_{j=1}^{K-1}\left(w_{2}+\lambda_{j}\right)\right]\right\} \prod_{j=1}^{K-1} \lambda_{j}}{\prod_{j=1}^{K-1}\left(w_{2}+\lambda_{j}\right)^{2}} d w_{2}
\end{aligned}
$$

Where $\mu=2^{2 R_{S}}$.

\subsection{Optimal power allocation}

Without losing the generality, it is assumed that the transmission total power of the relay node is 1 , if transmission power of the relay node $\mathrm{R}$ is $\alpha$, then the transmission power of the jammer node $\mathrm{J}$ is $1-\alpha$. The optimal power allocation problem to maximize the system secrecy capacity can be expressed as

$$
\alpha=\underset{0<\alpha<1}{\arg \max }\left\{C_{S}\right\}=\underset{0<\alpha<1}{\arg \max }\left\{\frac{1}{2} \log _{2}\left(\frac{1+\frac{\alpha\left|h_{\mathrm{RD}}\right|^{2}}{1+(1-\alpha)\left|h_{\mathrm{JD}}\right|^{2}}}{1+\frac{\alpha\left|h_{\mathrm{RE}}\right|^{2}}{1+(1-\alpha)\left|h_{\mathrm{JE}}\right|^{2}}}\right)\right\}
$$


Define $\left|h_{\mathrm{RD}}\right|^{2}=a,\left|h_{\mathrm{JD}}\right|^{2}=b,\left|h_{\mathrm{RE}}\right|^{2}=c,\left|h_{\mathrm{JE}}\right|^{2}=d$

substituting into (29) as:

$$
\alpha=\underset{0<\alpha<1}{\arg \max }\left\{\frac{1}{2} \log _{2}\left(\frac{[1+(1-\alpha) d][1+b+\alpha(a-b)]}{[1+(1-\alpha) b][1+d+\alpha(c-d)]}\right)\right\}
$$

The objective function of the optimization problem is expressed as

$$
f(\alpha)=\frac{[1+(1-\alpha) d][1+b+\alpha(a-b)]}{[1+(1-\alpha) b][1+d+\alpha(c-d)]}=\frac{\mathrm{A} \alpha^{2}+\mathrm{B} \alpha+\mathrm{C}}{\mathrm{D} \alpha^{2}+\mathrm{E} \alpha+\mathrm{F}}
$$

Where $\mathrm{A} \sim \mathrm{F}$ is unrelated coefficient with $\alpha$, The derivation of the objective function as

$$
f^{\prime}(\alpha)=\frac{(\mathrm{AE}-\mathrm{BD}) \alpha^{2}+2(\mathrm{AF}-\mathrm{DC}) \alpha+\mathrm{BF}-\mathrm{CE}}{\left(\mathrm{D} \alpha^{2}+\mathrm{E} \alpha+\mathrm{F}\right)^{2}}
$$

Define $f^{\prime}(\alpha)=0$, the optimal power distribution factor as

$$
\tilde{\alpha}=\frac{-2(\mathrm{AF}-\mathrm{DC})-\sqrt{\Delta}}{2(\mathrm{AE}-\mathrm{BD})}
$$

Where

$$
\Delta=4(\mathrm{AF}-\mathrm{DC})^{2}-4(\mathrm{AE}-\mathrm{BD})(\mathrm{BF}-\mathrm{CE})
$$

Normally, $\tilde{\alpha}$ are between 0 and 1 , system secrecy capacity is a convex function about $\alpha$, which is maximized when $\alpha=\tilde{\alpha}$. If $\tilde{\alpha}<0$, the system secrecy capacity is 0 , If $\tilde{\alpha}>1$ indicate that the power distribution factor of forwarding node $\mathrm{R}$ is 1 .

\section{SIMULATION RESULT AND DISCUSSION}

In this section, we compare performance between OS and OSJ by Monte Carlo simulation. Assuming that the transmission power of the source node and the relay node is equal $\left(P_{\mathrm{S}}=P_{\mathrm{R}}=P\right)$, For the OS scheme, the transmit power of the optimal forwarding node $\mathrm{R}$ is $P$. For the OSJ scheme, the power distribution factor $\alpha$ is assumed, the transmission power of forwarding node $\mathrm{R}$ is $P \alpha$, then the transmission power of jammer $\mathrm{J}$ is $(1-\alpha) P$.

Figure 2 shows system secrecy capacity performance of the optimal relay selection scheme(OS) and optimal relay selection with jamming scheme (OSJ) by formula (2) and (5). In the figure 2, the dotted and solid line indicates the system secrecy capacity when $K=4, K=6$ respectively. As shown in Fig. 2, firstly, when the number of relay increases, system secrecy capacity will be improved; secondly, the system secrecy capacity of the traditional optimal relay selection scheme is very slow with transmit power increasing, while the increase of system secrecy capacity is more obvious for optimal relay select with jamming when transmit power increase; thirdly, the selection of jammer $\mathrm{J}$ has a slight impact for the system secrecy capacity performance when the transmission power is low, while jammer $\mathrm{J}$ has a great impact when transmission power is high.
Figure 3 shows system secrecy outage probability performance with different transmission power $\mathrm{P}$ when $R_{\mathrm{S}}=0.1, R_{\mathrm{tr}}=2$. As shown in Fig. 2, firstly, optimal relay selection scheme with jamming(OSJ) has a lower secrecy outage probability compared with the optimal relay selection scheme(OS); secondly, secrecy outage probability for OS and OSJ scheme decrease with the increase of relay number; thirdly, when the transmission power is low, the impact for selection of cooperative interference node $\mathrm{J}$ is negligible, while transmission power is high, the system performance of secrecy outage probability for OSJ better compared with OS.

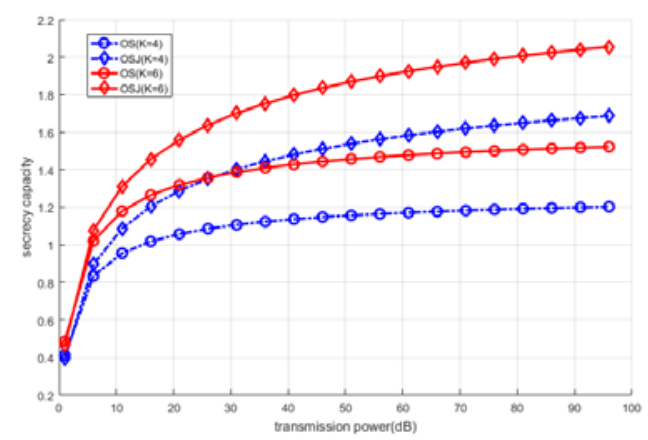

Figure 2: Secrecy capacity against the transmitted

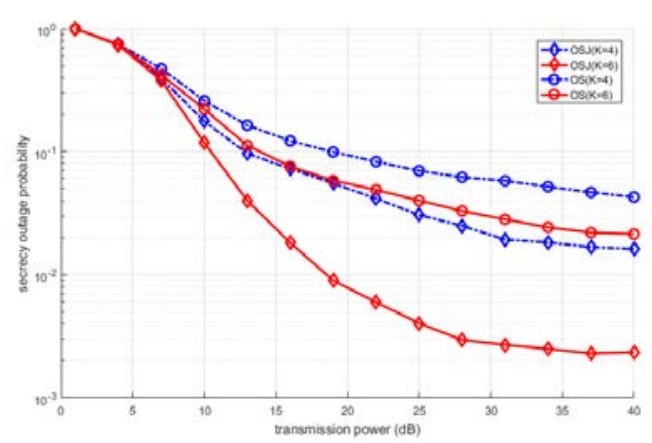

Figure 3: Secrecy outage probability against the transmitted

\section{CONCLUSIONS}

A joint optimal relay and jammer selection strategy is proposed based on the instantaneous channel state information of two-hop links in secure cooperative network. Firstly, an optimal relay node is selected to assist the source node forward information. Then another node is selected from the remaining relay nodes as the jammer to send artificial noise. Selection criteria of two schemes are given to maximize the system secrecy capacity by considering the channel state information for the legitimate users and the eavesdropper. The system secrecy outage probability progressive closed expressions are deduced, the optimal power distribution factor between jammer $\mathrm{J}$ and forwarding node $\mathrm{R}$ is given. It can be seen from the simulation results that the proposed joint relay and jammer selection scheme can significantly improve the system performance compared with the traditional relay selection method. 


\section{ACKNOWLEDGEMENTS}

This work was supported in part by the Graduate Student Scientific Research Innovation Ability Cultivation Plan Projects of Huaqiao University under the Grant 1611301036.

\section{REFERENCES}

[1] Papoulis, 2002. Probability. Random Variables, and Stochastic Processes, McGraw-Hill. New York, 4th edition.

[2] A.D.Wyner, 1975. The wiretap channel, Bell System Technical Journal. 54(8), pp.1355-1387.

[3] Csiszar and J. Korner, 1978. Broadcast channels with confidential messages, IEEE Transactions on Information Theory. 24(3), pp.339-348.

[4] Krikidis, 2010. Opportunistic relay selection for cooperative networks with secrecy constraints, IET Communications. 4(15), pp.1787-1791.

[5] Krikidis, J. S. Thompson and S. Mclaughlin, 2009. Relay selection for secure cooperative networks with jamming, IEEE Transactions on Wireless Communications. 8(10), pp.50035011.

[6] L. Dong, Z. Han, A. P. Petropulu and H. V. Poor, 2010. Improving wireless physical layer security via cooperating relays, IEEE Transactions on Signal Processing. vol. 58(3), pp.1875-1888.

[7] L. Fan, X. Lei, N. Yang, T. Q. Duong and G. K. Karagiannidis, 2017. Secrecy Cooperative Networks with Outdated Relay Selection Over Correlated Fading Channels, IEEE Transactions on Vehicular Technology. 66(8), pp.75997603.

[8] L. Tang and Q. Li, 2016. Wireless power transfer and cooperative jamming for secrecy throughput maximization, IEEE Wireless Communications Letters. 5(5), pp.556-559.

[9] L. Yang, J. Chen, H. Jiang, S. A. Vorobyov and H. Zhang, 2017. Optimal relay selection for secure cooperative communications with an adaptive eavesdropper, IEEE Transactions on Wireless Communications. 16(1), pp. 26-42.

[10] S. Leung-Yan-Cheong and M. Hellman, 1978. The Gaussian wire-tap channel, IEEE Transactions on Information Theory. 24(4), pp.451-456.

[11] Wei Zhou, Ruifeng Gao, Xiaodong Ji, Zhihua Bao, 2017. Hybrid secure transmission protocol for full - duplex relay system based on cooperative interference, Telecommunications Science. 33(1), pp.16-23.

[12] Weijia Lei, Meihui Zhan, Chengjing Xie, Xianzhong Xie, 2015. Physical layer security scheme based on jamming in energy cooperative network, Journal of Beijing University of Posts and Telecommunications. 38(6), pp. 65-68. 